\title{
Hohes Risiko durch ungeimpfte Ärzte und Pflegekräfte
}

\begin{abstract}
— Ungeimpfte Ärzte und Pflegekräfte sind in Deutschland ein großes Problem. Das wurde bei einer Podiumsdiskussion auf dem 123. Kongress der Deutschen Gesellschaft für Innere Medizin (DGIM) am 1. Mai 2017 in Mannheim deutlich.

Menschen in medizinischen Berufen sind häufig nicht geimpft und gefährden damit ihre Patienten. So waren in einer aktuellen Umfrage unter 1.200 medizinisch Beschäftigten in der Grippesaison nur 56\% der Ärzte und 35\% der Pflegekräfte gegen Influenza geimpft, wie der Präsident des Robert Koch-Instituts (RKI), Prof. Dr. Lothar Wieler, berichtete. Viele Gründe für fehlenden Schutz wurden genannt, außer "vergessen" und "Zweifel an der Wirksamkeit" aber auch die völlig unbegründete Furcht, „die Impfung könne Grippe auslösen", sagte Wieler. Fehlender Impfschutz bei medizinischem Personal kann sich dabei auch fatal auswirken, wie bei den Lahn-Dill-Kliniken in Wetzlar Anfang des Jahres. Dort waren Mitte Februar Masern bei einem Arzt festgestellt worden, der sich wahrscheinlich bei
\end{abstract}

\begin{abstract}
einem Patienten angesteckt hatte. Der Mediziner hatte in den zwei Tagen vor Diagnose Kontakt zu 130 Patienten gehabt, berichtete Dr. Norbert Köneke, Medizinischer Direktor der Klinik. In Folge wurden die Impfdokumente der 1.680 Beschäftigten überprüft und Mitarbeiter ohne sicheren Masernschutz vorübergehend nach Hause geschickt. „Den Klinikbetrieb in dieser Zeit aufrecht zu erhalten, war die größte Herausforderung", betonte Köneke.

Das Fazit: Es gab neun Mitarbeiter mit Masern, zum Glück aber nur einen Patienten mit Hinweisen auf nosokomiale Masern. Bei Laborkontrollen fanden sich 64 Mitarbeiter ohne schützende Antikörpertiter, 62 hätten sich inzwischen impfen lassen, bei zweien war die Lebendimpfung kontraindiziert. „Wir mussten hier nicht viel Überzeugungsarbeit leisten", betonte Köneke.

Kliniken könnten künftig auch rechtliche Auseinandersetzungen bei Masern drohen, die nachweislich von ungeimpften Mitarbeitern verbreitet wurden, warnte die Fachanwältin für Medizinrecht, Dr. Maike-Tjada
\end{abstract}

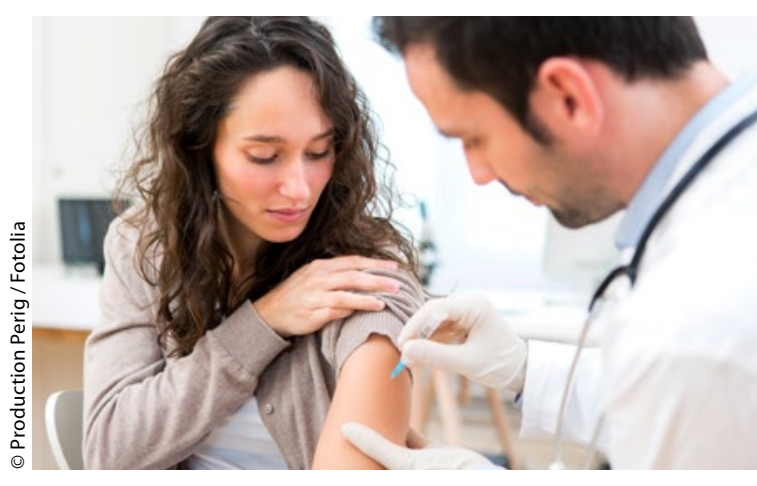

Mit dem Impfschutz ist es bei den medizinischen Berufen selbst nicht zum Besten bestellt.

Müller aus Mannheim. Arbeitgeber seien zudem in der Pflicht, medizinischem Personal die von der STIKO empfohlenen Impfungen anzubieten, ergänzte die Arbeitsmedizinerin Prof. Dr. Sabine Wicker vom Uniklinikum Frankfurt am Main. Bei Untätigkeit könne im Schadensfall Organisationsverschulden vorgeworfen werden.

(wg)

\section{Forschungsprojekt$$
\text { empCARE: Entlastung durch Zuwendung }
$$

— Zuwendung, Einfühlsamkeit und Aufmunterung sind Erwartungen an Pflegende, wenn sie mit Leiden oder starken Gefühlen von Patienten konfrontiert sind. Der stetige Umgang mit den Gefühlen der Patienten kann Pflegende aber auch psychisch belasten. „Pflege für Pflegende: Entwicklung und Verankerung eines empathiebasierten Entlastungkonzepts in der Care-Arbeit", kurz: empCare, heißt das Forschungsprojekt, an dem die Uniklinik Köln beteiligt ist. Hierbei geht es darum, ein Gesundheitspräventionskonzept für Pflegekräfte zu entwickeln und sie frühzeitig zu entlasten. „empCARE hilft unseren Pflegenden, bewusster mit ihren empathischen Fähigkeiten umzugehen und die Selbstpflege dabei nicht zu vernachlässigen. So entwickeln wir die Patientenversorgung weiter und stärken gleichzeitig die Pflegenden", so Vera Lux, Pflegedirektorin und Vorstandsmitglied der Uniklinik Köln. Bei der ersten Fachtagung zum Projekt empCARE am 18. Mai 2017 wurden erste Erfahrungen mit den Trainingsmaßnahmen für die Pflegenden vorgestellt und diskutiert.

Im Rahmen des Forschungsprogramms „Präventive Maßnahmen für die sichere und gesunde Arbeit von morgen" des Bildungsministeriums für Bildung und Forschung (BMBF) wird empCARE über eine Laufzeit von 42 Monaten gefördert. Die Fördersumme liegt bei insgesamt 900.000 Euro.

www.uk-koeln.de

\section{Firmenlauf 2017}

\section{Gemeinsam aktiv}

_ Um 18.30 Uhr fiel am 17. Mai 2017 der Startschuss für 6.000 Läufer auf der Waldau in Stuttgart - darunter auch 45 Pflege- und Verwaltungskräfte der Evangelischen Heimstiftung (EHS). Sie nahmen am 10. DEE-AOK Firmenlauf teil. Bereits zum sechsten Mal waren die EHS-Mitarbeiter auf der etwa sechs Kilometer langen Strecke dabei. Der Firmenlauf wird als gemeinsame Aktivität unter den Beschäftigten geschätzt. Zudem ist es eine Chance, auf die Bedeutung und auf die Realität von Pflegeberufen hinzuweisen. „Pflege ist ein bewegender Beruf, im tatsächlichen und übertragenen Sinn", sagt Bernhard Schneider, Hauptgeschäftsführer der EHS. „Zum einen bekommt man als Pfleger eine bewegende Wertschätzung und Dankbarkeit von den Bewohnern zurück. Zum anderen läuft man viele Kilometer am Tag durch die Einrichtung."

www.ev-heimstiftung.de 